\title{
Desenvolvimento Socioeconômico nas microrregiões do Paraná: uma análise multivariada
}

\author{
Vinícius Gonçalves Vidigal ${ }^{1}$ \\ Isis de Castro Amaral ${ }^{2}$ \\ Glauber Flaviano Silveira ${ }^{3}$
}

\begin{abstract}
Resumo: Este trabalho teve como objetivo avaliar as diferenças de nível de desenvolvimento socioeconômico entre as microrregiões do Estado do Paraná, bem como hierarquizá-las e, posteriormente, agrupá-las de acordo com suas principais características. Utilizou-se para tanto a análise estatística multivariada. Os principais resultados demonstraram a existência de disparidades regionais, a partir de indicadores de condições de moradia, de precariedade dos serviços de saúde e de desenvolvimento industrial, obtidos a partir da análise fatorial. A análise de cluster distribuiu as microrregiões em cinco grupos, sendo que o Grupo 2, formado pelos municípios de Cerro Azul e Pitanga, foi aquele com as piores condições de desenvolvimento. Todos os demais grupos apresentaram resultado negativo em apenas um dos três indicadores. Portanto, observa-se que coexistem carências extremamente importantes e que afetam milhares de famílias em todo o Estado do Paraná.
\end{abstract}

Palavras-chave: desenvolvimento socioeconômico; estatística multivariada; Paraná.

JEL: O18.

\footnotetext{
1 Mestre em Economia Aplicada pela Universidade Federal de Viçosa (UFV). Professor do Departamento de Economia da Universidade Estadual do Paraná (Unespar). E-mail: viniciusgv@hmail.com

2 Mestranda em Economia Aplicada pela Universidade Federal de Viçosa (UFV). Professora da FACIG. E-mail: isisamaral@yahoo.com.br

3 Mestre em Economia pela Universidade Federal de Viçosa (UFV). Economista da Fundação João Pinheiro (FJP). E-mail: glauber.economia@hotmail.com
} 


\title{
Socioeconomic Development in the regions of Paraná: a multivariate analysis
}

\begin{abstract}
This study had as objective to evaluate the differences in socioeconomic development levels among regions of Paraná State, as well as rank and then group them according to their main characteristics. The methodological tools refer to techniques of multivariate statistical analysis which were factor and cluster analysis. The main results showed the existence of great regional differences, considering indicators of housing conditions, precarious health services and industrial development, all obtained from the factor analysis. The cluster analysis distributed the regions in five groups, in which Group 2 was the one with the worst conditions of development. All other groups showed a negative result in only one of the three indicators. Therefore, it is observed that exist extremely important needs that affect thousands of families throughout the state of Paraná.
\end{abstract}

Key-words: socioeconomic development; multivariate statistics; Paraná. JEL: 018 .

\section{Introdução}

Um dos focos de estudo do campo de pesquisa em Economia Regional, desde sua origem, têm sido as desigualdades regionais. Tendo sido constatadas a existência de especificidades entre regiões, muito se discute a necessidade de programas sociais e políticas públicas que as levem em consideração. Como ressaltam Tavares e Porto Junior (2008), uma vez que a estruturação de políticas públicas deve ser ponderada pelas disparidades regionais, faz-se relevante determinar em quais aspectos as regiões do país se diferem ou se assemelham.

Segundo Rosado et al. (2009), a maior parte dos estudos que tratam das condições de vida de populações urbanas se restringem a considerar o baixo desenvolvimento como decorrência exclusiva da insuficiência de renda, pois as demais carências seriam eliminadas a partir de seu crescimento. No entanto, além da renda, devem-se considerar outros aspectos relacionados à qualidade de vida das populações. Dessa forma, os mesmos autores assinalam que uma abordagem alternativa do desenvolvimento é aquela que envolve também condições de acesso a serviços de infra-estrutura urbana, de saúde e de educação, adequação do consumo, nível de conforto domiciliar, entre outros.

O Estado do Paraná, objeto de estudo deste artigo, se encontra entre os mais desenvolvidos do país, mas apresenta fortes diferenças econômicas e sociais regionais no seu território (PNUD, 2010). Tais diferenças devemse, em parte, às importantes transformações ocorridas na base produtiva ao longo das últimas décadas. De acordo com Gualda e Tavares (2003), a 
agricultura cafeeira e a indústria pouco dinâmica deram lugar a um processo de reestruturação produtiva, que levou a um novo padrão de inserção e competição para a economia estadual. Além disso, as décadas de 1980 e 1990 foram marcadas por grandes reduções em investimentos em infraestrutura social, agravando a precariedade na oferta desses serviços por parte do setor público. Diante desse contexto, torna-se relevante estudar as principais diferenças existentes entre as microrregiões paranaenses, a partir de características relacionadas ao seu grau de desenvolvimento socioeconômico. Portanto, buscou-se avaliar as diferenças de grau de desenvolvimento socioeconômico entre as microrregiões do Estado do Paraná, com base em um conjunto de indicadores socioeconômicos. Ademais, procurou-se estabelecer uma hierarquização entre essas microrregiões e, posteriormente, agrupá-las de acordo com suas características.

O trabalho encontra-se estruturado em mais três seções, além desta introdução. A segunda seção apresenta os procedimentos metodológicos, baseados na análise estatística multivariada. Os resultados obtidos são apresentados e discutidos na terceira seção. Por fim, na quarta seção, são tecidas as considerações finais.

\section{Metodologia}

A escolha pela análise multivariada justifica-se por se referir, segundo Tavares (2008), a um conjunto de métodos estatísticos que tornam possível a análise simultânea de medidas múltiplas para cada indivíduo, objeto ou fenômeno observado. Logo, esta técnica é capaz de determinar o desempenho conjunto das variáveis analisadas, bem como especificar a importância de cada uma delas, na presença das demais.

A análise multivariada parece ser a técnica adequada a este estudo, tendo em vista que sua utilização tem se mostrado eficaz no tratamento de grande conjunto de informações. Ademais, seu emprego mostra-se comum em estudos sobre economia regional. Na literatura nacional, predominam os trabalhos que utilizaram tais técnicas em abordagens voltadas a aspectos de desenvolvimento econômico. Alguns exemplos são as análises de: Reis e Lima (1995) e Soares et al. (1999), para o Estado do Ceará; Perobelli et al. (1999) e Rosado et al. (2009), para regiões de Minas Gerais; Melo e Parré (2006; 2007), para regiões do Paraná; Tavares (2008), para o Sul do Brasil; e Leite et al. (2009), para os países do G-20.

A análise estatística multivariada tem servido também a várias outras linhas de estudo, tais como: estimação de degradação ambiental, com os casos de Silva e Ribeiro (2004) e de Fernandes et al. (2005); análise de distribuição de recursos públicos, realizada por Silva (1995); avaliações de desenvolvimento e 
de modernização na agropecuária, conduzidas por Hoffmann (1992), Ferreira Júnior et al. (2003) e Rezende e Parré (2003; 2004); entre outras.

Dado que o objetivo deste estudo é analisar as condições de moradia, industrialização, urbanização, infra-estrutura e saúde das microrregiões do Estado do Paraná, conduziu-se a análise fatorial pelo método de componentes principais com base em observações de vinte variáveis concernentes às questões de análise. De acordo com Haddad (1989), tal método é amplamente utilizado por reunir regiões segundo suas similaridades e agrupar as variáveis como forma de descrever padrões de variações. Obtém-se, assim, um conjunto de fatores que possibilita identificar o nível de desenvolvimento econômico, social e urbano de uma dada região.

Posteriormente, com o objetivo de classificar as microrregiões em razão dos escores fatoriais encontrados, foi empregada uma análise de cluster, ou agrupamento, que permitiu uma definição mais específica das características de cada microrregião. Conforme afirmam Fernau e Samson (1990), essa análise agrupa as microrregiões de forma a se ter variância mínima entre as mais semelhantes (homogeneidade máxima dentro do grupo) e variância máxima entre os grupos (heterogeneidade máxima entre grupos). Ambos os métodos, de análise fatorial e de cluster, são abordados de maneira mais detalhada nas seções a seguir.

\subsection{Análise Fatorial}

De acordo com Melo e Parré (2007), a análise fatorial tem como princípio básico a redução do número original de variáveis, por meio da extração de fatores independentes, de forma que estes possam explicar, de forma simples e reduzida, as variáveis originais.

O método de análise fatorial permite a obtenção de tantos fatores quanto for o número de variáveis originais, mas, normalmente, um número reduzido de fatores é suficiente para explicar uma elevada proporção da variância, ou seja, tal método consiste em obter fatores que são combinações lineares das variáveis originais (Johnson e Wichern, 1988; Rosado et al., 2009). A análise fatorial pode ser realizada através do método de componentes principais, na qual o primeiro fator contém o maior percentual de explicação da variância total das variáveis da amostra; o segundo fator contém o segundo maior percentual e assim por diante. Tal técnica foi empregada neste estudo e considerou-se um número máximo de fatores que explicam, conjuntamente, 75\% da proporção da variância.

O método de análise fatorial pode ser expresso na forma matemática através de uma combinação linear entre as variáveis $\left(X_{i}\right)$ e $k$ fatores comuns $(F)$ :

$$
X_{i}=A_{i 1} F_{1}+A_{i 2} F_{2}+\ldots+A_{i k} F_{k}+U_{i}+E_{i}
$$


em que $A_{i k}$ representa as cargas fatoriais, usadas para combinar linearmente os fatores comuns; $F_{k}$, os fatores comuns; $U_{i}$, o fator único; e $E_{i}$, o erro aleatório da estimativa.

As cargas fatoriais indicam a intensidade das relações entre as variáveis normalizadas $X_{i}$ e os fatores. Visando simplificar as comparações entre as variáveis e as observações, é prudente expressar estas em termos de desviopadrão, isto é, deve-se normalizar as variáveis e as observações em relação a sua média (Mingoti, 2007). Quanto maior uma carga fatorial, maior a associação da variável com o fator. A variância comum $h_{i}^{2}$, ou comunalidade, representa quanto da variância total de $X_{i}$ é reproduzida pelos fatores comuns, sendo calculada a partir do somatório ao quadrado das cargas fatoriais. A comunalidade permite verificar se o modelo com $n$ fatores representa bem as variáveis originais. Assim, um primeiro fator é escolhido para maximizar a soma dos quadrados das cargas fatoriais em relação a ele. Em seguida, obtém-se um segundo fator, para que também seja maximizada a soma de quadrados das cargas fatoriais, e assim por diante. A comunalidade pode ser expressa como segue:

$$
h_{i}^{2}=a_{i 1}^{2}+a_{i 2}^{2}+a_{i 3}^{2}+\ldots+a_{i f}^{2}
$$

Em relação aos demais componentes da equação 1, tem-se que a variância única $U_{i}$ é a parte da variância total que não se associa com a variância das outras variáveis. Já o termo $E_{i}$ representa o erro de observação, de mensuração ou de especificação do modelo. $U_{i}+E_{i}$ caracteriza a unicidade $\left(d_{i}^{2}\right)$ e pode ser obtido fazendo-se $\left(1-h_{i}^{2}\right)$. Dentre as propriedades do método de análise fatorial, merece destaque o que se refere a $\mathrm{E}\left(E_{i}\right)=\mathrm{E}(F)=0$; e à ortogonalidade dos fatores. A equação abaixo demonstra a unicidade:

$$
d_{i}^{2}=U_{i}^{2}+e_{i}^{2}
$$

A medida que expressa a variância total do modelo explicada por cada fator consiste no autovalor ou raiz característica. De acordo com Mingoti (2007), na determinação do número de fatores necessários para representar o conjunto de dados, usualmente consideram-se apenas os fatores cuja raiz característica é maior que a unidade. E o seu valor é o somatório dos quadrados das cargas fatoriais de cada variável associadas ao fator específico. Por fim, a razão entre o autovalor e o número de variáveis $\left(X_{i}\right)$ determina a proporção da variância total explicada pelo fator.

Para facilitar a interpretação dos fatores há a possibilidade de se realizar uma rotação de fatores, a qual procura minimizar o número de variáveis fortemente relacionadas com cada fator, permitindo, assim, obter fatores mais interpretáveis (Mingoti, 2007). Uma vez que os vetores característicos não são únicos, as cargas fatoriais da análise fatorial por componentes principais podem ser modificadas sem que haja prejuízo à análise. A rotação de fatores consiste em modificar as cargas fatoriais, a partir do cálculo de uma nova 
matriz $A$, com o objetivo de obter resultados de mais fácil interpretação e cujos fatores se relacionem mais distintamente com certo grupo de variáveis.

Após a obtenção das cargas fatoriais, determinam-se os escores fatoriais associados aos fatores obtidos após a rotação ortogonal dos fatores. Os escores fatoriais são valores estimados para cada fator em cada observação, com o objetivo de situá-las no espaço dos fatores comuns. Para isso, a matriz de coeficientes fatoriais foi estimada a partir da multiplicação da matriz inversa de correlação pela matriz de cargas fatoriais. A partir dessa multiplicação, foram calculados os escores fatoriais para cada microrregião do Estado do Paraná, de modo a possibilitar uma hierarquização e a implementação da análise de cluster.

No modelo de análise fatorial, há uma medida de adequação dos dados, o Kaiser-Meyer-Olkin Measure of Sampling Adequacy (KMO), que se refere à razão entre a soma dos quadrados das correlações de todas as variáveis e essa mesma soma acrescentada da soma dos quadrados das correlações parciais de todas as variáveis. Logo, o KMO é um teste que examina o ajustamento dos dados, tomando todas as varáveis simultaneamente, e que provê uma informação sintética sobre os mesmos. Ademais, o KMO varia entre zero e um, sendo que para a interpretação desse teste considera-se: 0,9-1, como excelente; o,8-0,9, ótimo; 0,7-0,8, bom; 0,6-0,7, regular; 0,5-0,6, ruim; $0-0,5$, inadequado. Outro teste, que precede a análise fatorial com vistas à verificação de suas premissas, é o Bartlett Test of Sphericity (BTS), o qual testa a hipótese de que a matriz de correlação é uma matriz identidade, ou seja, de que não há correlação entre as variáveis.

\subsection{Análise de Chuster}

As análises de cluster admitem abordagens hierárquicas e não hierárquicas, sendo que este estudo mantém enfoque sobre a primeira. Consideram-se duas possibilidades: a aglomerativa e a divisível. A aglomerativa considera que de início cada indivíduo se encontra isolado, como que formando um "cluster individual". O processo segue aproximando esses indivíduos de acordo com suas similaridades até que se atinja uma estabilidade relativa, que variará em função dos objetivos de análise. A abordagem divisível considera que se opera em sentido contrário, "desaglomerando" um cluster único inicial. Neste estudo, a opção empregada é a abordagem hierárquica aglomerativa. Os agrupamentos serão, então, representados graficamente por um dendograma que mostra, a um tempo, as possíveis aglomerações e sua consistência relativa, a qual varia em função da "proximidade" entre os indivíduos em termos da distância euclidiana.

No método hierárquico aglomerativo, os critérios de agrupamento mais utilizados são o da associação simples, que é baseada nas menores distâncias 
entre os objetos, e o da associação completa, baseado na maior distância entre os objetos. Cada solução de cluster gerada deve ser devidamente interpretada, a fim de que se identifique a mais adequada para dar um significado aos dados em questão (Rosado et al., 2009).

A obtenção dos agrupamentos exige que se estime uma medida de similaridade ou dissimilaridade entre os objetos a serem agrupados e, depois, se adote uma técnica de agrupamento para a formação dos grupos (Rosado et al., 2009). Os algoritmos utilizados para tal fim baseiam-se na quantificação da distância entre agrupamentos, destacando-se: o método de Ward (variância mínima); o método de ligação simples, ou do vizinho mais próximo; o método de ligação completa, ou do vizinho mais distante; o método do centróide; e o método da mediana. Neste estudo, o método de Ward apresentou os melhores resultados e foi, portanto, utilizado. Seu objetivo consiste em minimizar o quadrado da distância euclidiana às médias dos conglomerados, com distância euclidiana quadrática dada por:

$$
D_{a b}=j=\Sigma_{n}\left(X_{a j}-X_{b j}\right)^{2}
$$

em que o somatório vai de 1 até $n$ (numero de variáveis); e $a$ e $b$ são os objetos analisados, podendo se generalizar para todos os elementos do estudo.

\subsection{Variáveis e Fonte de Dados}

No que diz respeito ao critério para escolha das variáveis de análise, parece não existir um consenso quanto a essa determinação em trabalhos acerca de desigualdades regionais. Dado isto, não se pode afirmar que existe uma medida simples e consensual de desempenho econômico e social de uma região, mas que coexistem inúmeras alternativas de mensuração, tais como PIB, PIB per capita, rendimento domiciliar total, crescimento demográfico, fatores relacionados à infraestrutura de transportes, indicadores de proximidade geográfica, entre outros.

Logo, considerando uma tentativa de exprimir variáveis que dimensionem a realidade socioeconômica das microrregiões de um estado, bem como a desigualdade, é que este estudo se apóia numa base de dados composta por vinte variáveis, centradas, principalmente, em aspectos estritamente sociais.

Foram obtidos valores para as 39 microrregiões do Estado do Paraná, as quais são apresentadas na Figura A1 do Anexo. As variáveis selecionadas, que se referem ao ano de 2000, foram: analf, pessoas analfabetas (\%); clixo, domicílios com coleta de lixo (\%); sagua, domicílios com água não canalizada (\%); sban, domicílios sem banheiro (\%); rendmin, domicílios em que a pessoa responsável tinha rendimento mensal de até $1 / 4$ de salário mínimo (\%); gelad, domicílios que possuíam geladeira (\%); ilumin, domicílios com iluminação 
elétrica (\%); comput, domicílios que possuíam microcomputadores (\%); tel, domicílios com linha telefônica (\%); telev, domicílios que possuíam televisão (\%); leit, $\mathrm{n}^{\circ}$ de leitos hospitalares para cada cem habitantes; hosp, $\mathrm{n} .{ }^{\circ}$ de estabelecimentos de saúde para cada mil habitantes; ddemog, densidade demográfica (hab/ $\mathrm{km}^{2}$ ); urban, taxa de urbanização (\%); morad, número médio de moradores por domicílio; mort, taxa de mortalidade (\%); pibagro, PIB da agropecuária (\% do PIB total); pibind, PIB da indústria (\% do PIB total); pibserv, PIB de serviços (\% do PIB total); e pibcapita, PIB per capita.

Os dados referentes às variáveis utilizadas são secundários e provenientes do Censo Demográfico de 2000, os quais foram obtidos por meio do Sistema IBGE de Recuperação Automática (SIDRA) do IBGE (2010). De forma a se ter uma análise preliminar das variáveis e das microrregiões analisadas, são apresentadas a seguir estatísticas descritivas.

TABELA 1 . MÉDIA, DESVIO PADRÃO, VALORES MÍNIMOS E MÁXIMOS DAS VARIÁVEIS SOCIOECONÔMICAS - MICRORREGIÕES PARANAENSES

\begin{tabular}{lrrrr}
\hline Variável & Média & $\begin{array}{c}\text { Desvio } \\
\text { padrão }\end{array}$ & $\begin{array}{c}\text { Valor } \\
\text { mínimo }\end{array}$ & $\begin{array}{c}\text { Valor } \\
\text { máximo }\end{array}$ \\
\hline analf & 13,29 & 3,79 & 7,20 & 24,25 \\
clixo & 71,42 & 16,37 & 29,39 & 96,85 \\
sagua & 4,35 & 5,03 & 0,15 & 19,11 \\
sban & 3,32 & 4,16 & 0,16 & 23,13 \\
rendmin & 0,33 & 0,23 & 0,03 & 1,20 \\
gelad & 85,51 & 9,76 & 52,71 & 96,76 \\
ilumin & 95,89 & 5,36 & 74,74 & 99,79 \\
comput & 6,64 & 3,63 & 2,10 & 19,54 \\
tel & 28,79 & 14,61 & 8,52 & 66,02 \\
telev & 85,69 & 9,12 & 44,77 & 94,00 \\
leit & 0,27 & 0,10 & 0,06 & 0,47 \\
hosp & 0,54 & 0,12 & 0,32 & 0,76 \\
ddemog & 50,18 & 65,54 & 8,50 & 309,95 \\
urban & 69,94 & 17,17 & 21,20 & 96,13 \\
morad & 3,30 & 0,12 & 3,10 & 3,60 \\
mort & 0,51 & 0,12 & 0,31 & 0,79 \\
pibagro & 0,21 & 0,11 & 0,01 & 0,56 \\
pibind & 0,23 & 0,11 & 0,05 & 0,59 \\
pibserv & 0,56 & 0,09 & 0,36 & 11,30 \\
pibcapita & 5,48 & 1,95 & 2,82 &
\end{tabular}

FONTE: resultados de pesquisa. 
A observação dos valores mínimos e máximos chama a atenção para a microrregião de Cerro Azul. É evidente que se trata de uma região com condições econômicas e sociais muito inferiores ao restante do estado, visto que dos vinte indicadores selecionados, essa região responde pelo pior nível em treze deles (analf, clixo, sban, gelad, ilumin, comput, tel, telev, leit, ddemog, urban, pibagro e pibind). A esse respeito, pode-se relacionar o fato de que Cerro Azul tem a agropecuária como principal setor da economia (56\% do PIB), uma vez que essa atividade não gera tanto valor agregado quanto as demais. De maneira semelhante, a microrregião de Pitanga se encontra entre as piores microrregiões para oito indicadores (rendmin, gelad, ilumin, tel, telev, hosp, urban e pibcapita), com destaque para sua última posição em nível de renda per capita.

Por outro lado, algumas microrregiões estiveram entre as melhores para a maior parte das variáveis, como é o caso de Maringá, Londrina e Curitiba. Contudo, essas três regiões se destacaram negativamente, junto a Cerro Azul, como aquelas com menor presença de estabelecimentos de saúde por habitante. Finalmente, a microrregião de Foz do Iguaçu, apesar de apresentar vários bons indicadores, é aquela com maior taxa de mortalidade. Essa condição é resultado do elevado índice de homicídios que, inclusive, caracteriza-a como uma das regiões mais violentas do país.

\section{Resultados}

Um passo que precede a aplicação da análise fatorial refere-se à condução de testes que verifiquem a aplicabilidade desse método aos dados da amostra. O exame da matriz anti-imagem (Tabela A2, Anexo), que contém o negativo das correlações parciais, demonstra que a análise fatorial seria adequada, pois os coeficientes nela contidas apresentaram valores baixos.

Outro importante critério é o teste de esfericidade de Bartlett (BTS), com o objetivo de testar a significância de pelo menos algumas das correlações entre as variáveis. A estatística do teste resultou em um valor de 1.222,90, o qual foi significativo ao nível de $1 \%$ de significância e levou à rejeição da hipótese nula de que a matriz de correlação fosse igual a uma matriz identidade.

Um último teste empregado na verificação da adequabilidade da análise fatorial ao conjunto de dados das microrregiões paranaenses foi o de KaiserMeyer-Olkin (KMO). O valor da estatística para o conjunto das variáveis foi de 0,724 , ou seja, indicou que as mesmas são apropriadas para a aplicação da análise fatorial (Tabela A1, Anexo).

A análise fatorial permitiu a obtenção de quatro fatores com autovalores superiores à unidade, os quais resumem as informações contidas nas 
vinte variáveis originais. Os resultados, após a rotação fatorial, mostram que esses quatro fatores explicam aproximadamente $82,46 \%$ da variância total dos dados. O Fator 1 é o que possui maior percentual de variância explicada (34,84\%). Os três primeiros fatores, analisados conjuntamente, são responsáveis por 75,06\%, sendo assim os mais representativos (Tabela 2).

TABELA 2. AUTOVALORES, VARIÂNCIA EXPLICADA PELOS FATORES E VARIÂNCIA ACUMULADA

\begin{tabular}{lccc}
\hline Fator & Autovalores & $\begin{array}{c}\text { Variância explicada } \\
(\%)\end{array}$ & $\begin{array}{c}\text { Variância acumulada } \\
(\%)\end{array}$ \\
\hline Fator 1 & 6,97 & 34,84 & 34,84 \\
Fator 2 & 4,20 & 21,01 & 55,85 \\
Fator 3 & 3,84 & 19,21 & 75,06 \\
Fator 4 & 1,48 & 7,40 & 82,46 \\
\hline
\end{tabular}

FONTE: resultados de pesquisa.

O método de rotação que apresentou os melhores resultados foi o de rotação ortogonal Varimax. A Tabela 3 traz as cargas fatoriais e comunalidades resultantes para cada variável. De modo a apresentar mais claramente as correlações entre os fatores e as variáveis selecionadas, considerou-se, para interpretação, as cargas fatoriais superiores a 0,50 (destacadas em negrito). Percebe-se que o Fator 1 ( $\mathrm{F} 1)$ relaciona-se forte e positivamente com as variáveis clixo (proporção de domicílios com coleta de lixo), gelad (proporção de domicílios com geladeira), ilumin (proporção de domicílios com iluminação elétrica), telev (proporção de domicílios com televisão) e urban (taxa de urbanização); ao passo que tem correlação alta e negativa com sagua (proporção de domicílios com água não-canalizada), sban (proporção de domicílios sem banheiro), rendmin (proporção de domicílios com rendimento até $1 / 4$ de salário mínimo) e morad (média de moradores por domicílio). Observa-se que todas as variáveis são relacionadas à infra-estrutura de habitação da população e à urbanização nas microrregiões paranaenses, de forma que as condições de moradia serão tão melhores quanto maior for o valor do F1.

As variáveis que predominam no Fator (F2) são clixo (proporção de domicílios com coleta de lixo), comput (proporção de domićlios com microcomputadores), tel (proporção de domicílios com linha telefônica), ddemog (densidade demográfica), urban (taxa de urbanização) e pibserv (participação do setor de serviços no PIB), com correlações altas e positivas; e leit (número de leitos hospitalares por habitante), hosp (número de estabelecimentos de saúde por habitante) e pibagro (participação da agropecuária no PIB), com correlações negativas. Logo, verifica-se que o F2 se relaciona com aspectos referentes à precariedade (insuficiência) dos serviços de saúde associados ao fenômeno 
da urbanização dos municípios. Dessa forma, quanto maior for esse fator, piores serão os aspectos de infra-estrutura de saúde.

TABELA 3. CARGAS FATORIAIS E COMUNALIDADES OBTIDAS PELA ANÁLISE FATORIAL, APÓS ROTAÇÃO PELO MÉTODO VARIMAX

\begin{tabular}{|c|c|c|c|c|}
\hline Variável & Fator 1 & Fator 2 & Fator 3 & Comunalidade \\
\hline analf & $-0,307$ & $-0,187$ & $-0,726$ & 0,656 \\
\hline clixo & 0,744 & 0,522 & 0,267 & 0,897 \\
\hline sagua & $-0,821$ & $-0,336$ & 0,091 & 0,794 \\
\hline sban & $-0,884$ & $-0,074$ & $-0,290$ & 0,872 \\
\hline rendmin & $-\mathbf{0 , 5 0 5}$ & $-0,338$ & $-0,386$ & 0,517 \\
\hline gelad & o,892 & 0,253 & 0,205 & 0,901 \\
\hline ilumin & 0,944 & 0,124 & 0,183 & 0,940 \\
\hline comput & 0,313 & 0,763 & 0,454 & 0,886 \\
\hline tel & 0,419 & o,659 & 0,479 & o,838 \\
\hline telev & $\mathbf{0 , 8 9 3}$ & 0,173 & 0,300 & 0,916 \\
\hline leit & 0,470 & $-\mathbf{0 , 5 3 7}$ & $-0,424$ & 0,689 \\
\hline hosp & $-0,229$ & $-0,741$ & 0,070 & 0,607 \\
\hline ddemog & 0,129 & $\mathbf{0 , 8 3 4}$ & 0,264 & 0,781 \\
\hline urban & 0,762 & $\mathbf{0 , 5 0 5}$ & 0,253 & 0,899 \\
\hline morad & $-0,728$ & $-0,005$ & 0,484 & 0,765 \\
\hline mort & $-0,131$ & $-0,001$ & 0,084 & 0,024 \\
\hline pibagro & $-0,434$ & $-0,509$ & $-0,581$ & 0,784 \\
\hline pibind & 0,073 & 0,066 & 0,908 & o,833 \\
\hline pibserv & 0,437 & 0,562 & $-0,414$ & 0,679 \\
\hline pibcapita & 0,228 & 0,413 & 0,714 & 0,733 \\
\hline
\end{tabular}

FONTE: resultados de pesquisa.

Por fim, o Fator 3 (F3) apresenta correlação elevada e positiva com os indicadores pibind (participação do PIB da indústria), pibserv (participação do PIB de serviços) e pibcapita (PIB per capita); ao passo que existe relação inversa entre esse fator e analf (taxa de analfabetismo) e pibagro (participação do PIB da agropecuária). Verifica-se, portanto, que esse fator representa o desenvolvimento industrial associado a melhores níveis de educação.

A etapa seguinte a análise das cargas fatoriais é a observação dos escores fatoriais, que correspondem aos valores calculados dos fatores para cada microrregião paranaense. Deve-se ressaltar que, em conjunto, os valores dos escores fatoriais das microrregiões possuem média zero e desvio padrão igual 
a um. Logo, como afirmam Melo e Parré (2007), uma possível interpretação consiste em considerar os escores com valores próximos de zero como indicadores de nível de desenvolvimento médio e aqueles maiores e mais distantes de zero como referentes a regiões mais avançadas.

A Tabela 4 traz a hierarquização das microrregiões do Paraná segundo as condições de moradia (F1). Observa-se que 61,5\% das microrregiões apresentaram valores positivos para $\mathrm{F} 1$, ou seja, têm as melhores condições no aspecto tratado por esse fator. As microrregiões com os mais altos valores são Floraí (1,456), Toledo (1,132), Umuarama (0,982) e Apucarana $(0,959)$. Por outro lado, as piores microrregiões foram São Mateus do Sul $(-1,273)$, Prudentópolis $(-1,728)$, Pitanga $(-2,686)$ e Cerro Azul $(-3,554)$.

TABELA 4. HIERARQUIZAÇÃO DAS MICRORREGIÕES PARANAENSES DE ACORDO COM O INDICADOR DE CONDIÇÕES DE MORADIA (FATOR 1)

\begin{tabular}{cll|clc}
\hline Posição & Microrregião & F1 & Posição & Microrregião & F1 \\
\hline 1 & Floraí & 1,456 & 21 & Capanema & 0,179 \\
2 & Toledo & 1,132 & 22 & Maringá & 0,177 \\
3 & Umuarama & 0,982 & 23 & Francisco Beltrão & 0,079 \\
4 & Apucarana & 0,959 & 24 & Lapa & 0,006 \\
5 & Porecatu & 0,786 & 25 & Ponta Grossa & $-0,157$ \\
6 & Cianorte & 0,784 & 26 & União da Vitória & $-0,162$ \\
7 & Cornélio Procópio & 0,773 & 27 & Jaguariava & $-0,166$ \\
8 & Astorga & 0,765 & 28 & Curitiba & $-0,193$ \\
9 & Jacarezinho & 0,749 & 29 & Ibaiti & $-0,208$ \\
10 & Paranavaí & 0,722 & 30 & Ivaiporá & $-0,392$ \\
11 & Faxinal & 0,700 & 31 & Irati & $-0,502$ \\
12 & Cascavel & 0,613 & 32 & Rio Negro & $-0,669$ \\
13 & Foza do Iguaçu & 0,549 & 33 & Palmas & $-0,675$ \\
14 & Pato Branco & 0,543 & 34 & Telêmaco Borba & $-0,697$ \\
15 & Goioerê & 0,443 & 35 & Guarapuava & $-0,794$ \\
16 & Wenceslau Braz & 0,370 & 36 & S. Mateus do Sul & $-1,273$ \\
17 & Campo Mourão & 0,344 & 37 & Prudentópolis & $-1,728$ \\
18 & Assaí & 0,338 & 38 & Pitanga & $-2,686$ \\
19 & Paranaguá & 0,219 & 39 & Cerro Azul & $-3,554$ \\
20 & Londrina & 0,189 & & & \\
\hline & & & &
\end{tabular}

FONTE: resultados de pesquisa. 
No que tange o Fator 2, os maiores escores foram obtidos pelas microrregiões de Londrina (2,552), Maringá $(2,708)$ e Curitiba (3,103), as quais se deparam com as piores condições em termos de oferta de serviços de saúde decorrente da urbanização. A oferta limitada desses serviços se explica pelo fato de se tratarem de regiões que passaram por processo de concentração populacional, impulsionado pela evasão ocorrida no meio rural nas últimas décadas (IPARDES, 2004). Esse é um fator que contribui, junto à escassez de investimentos, para a limitação no atendimento à população. As regiões de São Mateus do Sul $(-1,344)$, Palmas $(-1,165)$, Irati $(-0,985)$ e União da Vitória $(-0,913)$, por sua vez, são aquelas com melhor situação no que diz respeito a esse fator (Tabela 5).

\section{TABELA 5. HIERARQUIZAÇÃO DAS MICRORREGIÕES PARANAENSES DE ACORDO COM O INDICADOR DE URBANIZAÇÃO ASSOCIADA À PRECARIEDADE DOS SERVIÇOS DE SAÚDE (FATOR 2)}

\begin{tabular}{clc|clc}
\hline Posição & Microrregião & F2 & Posição & Microrregião & F2 \\
\hline 1 & S. Mateus do Sul & $-1,344$ & 21 & Astorga & $-0,103$ \\
2 & Palmas & $-1,165$ & 22 & Umuarama & $-0,053$ \\
3 & Irati & $-0,985$ & 23 & Jacarezinho & $-0,017$ \\
4 & União da Vitória & $-0,913$ & 24 & Wenceslau Braz & 0,049 \\
5 & Jaguariaíva & $-0,897$ & 25 & Ibaiti & 0,080 \\
6 & Francisco Beltrão & $-0,894$ & 26 & Assaí & 0,114 \\
7 & Floraí & $-0,860$ & 27 & Cornélio Procópio & 0,212 \\
8 & Rio Negro & $-0,848$ & 28 & Porecatu & 0,227 \\
9 & Prudentópolis & $-0,781$ & 29 & Campo Mourão & 0,254 \\
10 & Capanema & $-0,760$ & 30 & Foz do Iguaçu & 0,254 \\
11 & Lapa & $-0,708$ & 31 & Apucarana & 0,322 \\
12 & Toledo & $-0,614$ & 32 & Cerro Azul & 0,501 \\
13 & Goioerê & $-0,528$ & 33 & Cascavel & 0,550 \\
14 & Telêmaco Borba & $-0,491$ & 34 & Paranaguá & 0,645 \\
15 & Faxinal & $-0,467$ & 35 & Pitanga & 0,935 \\
16 & Ivaiporã & $-0,354$ & 36 & Ponta Grossa & 0,974 \\
17 & Pato Branco & $-0,351$ & 37 & Londrina & 2,552 \\
18 & Cianorte & $-0,261$ & 38 & Maringá & 2,708 \\
19 & Guarapuava & $-0,193$ & 39 & Curitiba & 3,103 \\
20 & Paranavaí & $-0,120$ & & & \\
\hline FONEa & & & \\
\hline
\end{tabular}

FONTE: resultados de pesquisa. 
A Tabela 6, que contém os escores fatoriais das microrregiões referentes ao Fator 3, mostra que as microrregiões de Foz do Iguaçu $(2,142)$, Palmas $(1,86)$ e São Mateus do Sul $(1,743)$ são aquelas com maior desenvolvimento industrial associado a melhores níveis de educação (menor analfabetismo) e que as piores microrregiões são Ivaiporã $(-1,351)$, Cerro Azul $(-1,437)$, Faxinal $(-1,601)$ e Pitanga $(-1,633)$. Faz-se interessante ressaltar que Cerro Azul e Pitanga apresentaram os valores mais baixos também para o F1.

TABELA 6. HIERARQUIZAÇÃO DAS MICRORREGIÕES PARANAENSES DE ACORDO COM O INDICADOR DE DESENVOLVIMENTO INDUSTRIAL ASSOCIADO A MELHORES NÍVEIS DE EDUCAÇÃO (FATOR 3)

\begin{tabular}{cll|lll}
\hline Posição & Microrregião & F3 & Posição & Microrregião & F3 \\
\hline 1 & Foz do Iguaçu & 2,142 & 21 & Cianorte & 0,005 \\
2 & Palmas & 1,860 & 22 & Pato Branco & $-0,029$ \\
3 & S. Mateus do Sul & 1,743 & 23 & Jacarezinho & $-0,087$ \\
4 & Rio Negro & 1,268 & 24 & Astorga & $-0,311$ \\
5 & Paranaguá & 1,241 & 25 & Paranavaí & $-0,465$ \\
6 & Ponta Grossa & 1,227 & 26 & Capanema & $-0,483$ \\
7 & Jaguariaíva & 1,214 & 27 & Cornélio Procópio & $-0,554$ \\
8 & Curitiba & 1,195 & 28 & Porecatu & $-0,658$ \\
9 & União da Vitória & 0,638 & 29 & Floraí & $-0,687$ \\
10 & Irati & 0,535 & 30 & Campo Mourão & $-0,703$ \\
11 & Lapa & 0,524 & 31 & Goioerê & $-1,027$ \\
12 & Londrina & 0,450 & 32 & Assaí & $-1,091$ \\
13 & Apucarana & 0,436 & 33 & Umuarama & $-1,146$ \\
14 & Guarapuava & 0,346 & 34 & Ibaiti & $-1,158$ \\
15 & Maringá & 0,304 & 35 & Wenceslau Braz & $-1,200$ \\
16 & Prudentópolis & 0,188 & 36 & Ivaiporã & $-1,351$ \\
17 & Francisco Beltrão & 0,125 & 37 & Cerro Azul & $-1,437$ \\
18 & Cascavel & 0,081 & 38 & Faxinal & $-1,601$ \\
19 & Toledo & 0,052 & 39 & Pitanga & $-1,633$ \\
20 & Telêmaco Borba & 0,046 & & & \\
\hline FONTE: resultados de pesquisa. & & & &
\end{tabular}

No intuito de se estabelecer agrupamentos entre as microrregiões paranaenses, posteriormente à hierarquização das mesmas, procedeu-se com a utilização da técnica multivariada da análise de cluster. Como ilustra o dendograma da Figura 1, o método de Ward apontou para a formação de cinco grupos, os quais foram formados a partir das características contidas nas variáveis em estudo. 
FIGURA 1. DENDOGRAMA DA ANÁLISE DE AGRUPAMENTO DOS ESCORES FATORIAIS DAS MICRORREGIÕES PARANAENSES - MÉTODO DE WARD

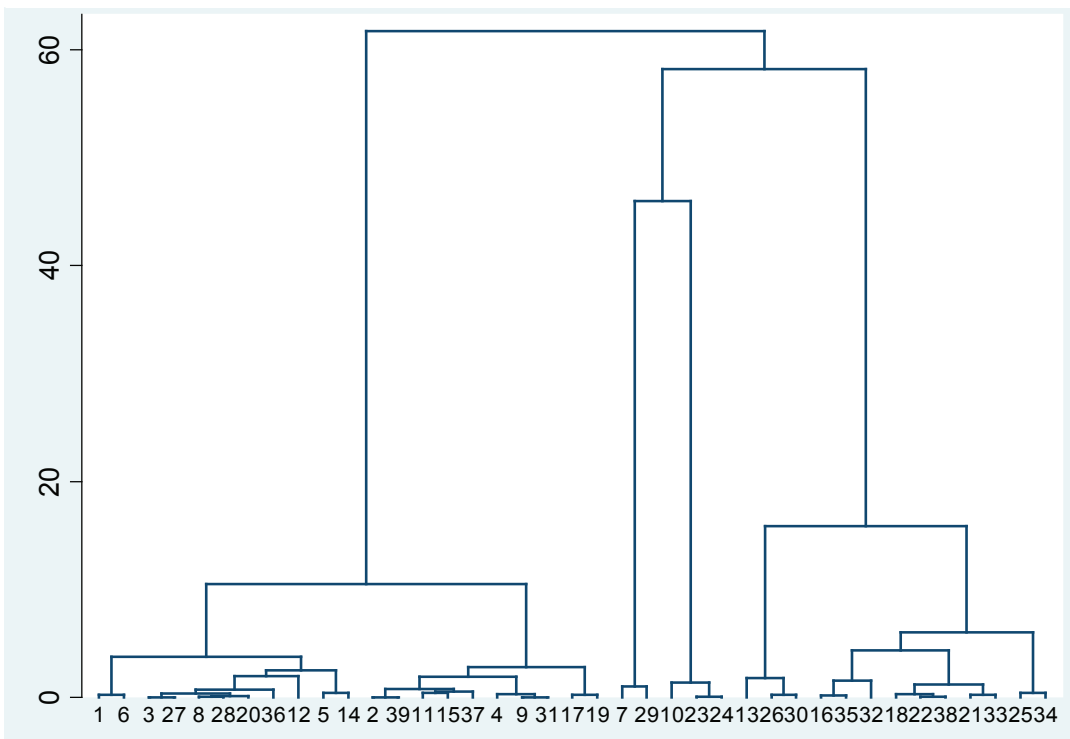

FONTE resultados de pesquisa.

Os cinco agrupamentos resultantes distribuíram as microrregiões como segue: Grupo 1: Apucarana, Assaí, Astorga, Campo Mourão, Capanema, Cascavel, Cianorte, Cornélio Procópio, Faxinal, Floraí, Francisco Beltrão, Goioerê, Ibaiti, Ivaiporã, Jacarezinho, Paranavaí, Pato Branco, Porecatu, Toledo, Umuarama e Wenceslau Braz; Grupo 2: Cerro Azul e Pitanga; Grupo 3: Curitiba, Londrina e Maringá; Grupo 4: Foz do Iguaçu, Paranaguá e Ponta Grossa; e Grupo 5: Guarapuava, Irati, Jaguariaíva, Lapa, Palmas, Prudentópolis, Rio Negro, São Mateus do Sul, Telêmaco Borba e União da Vitória.

A Figura 2 mostra a distribuição geográfica dos agrupamentos das microrregiões, na qual tem-se dois grandes blocos com significativa proximidade territorial entre a maior parte de seus componentes: o Grupo 1, abrangendo as partes Norte, Noroeste e Oeste; e o Grupo 5, concentrado principalmente no sul do estado. 


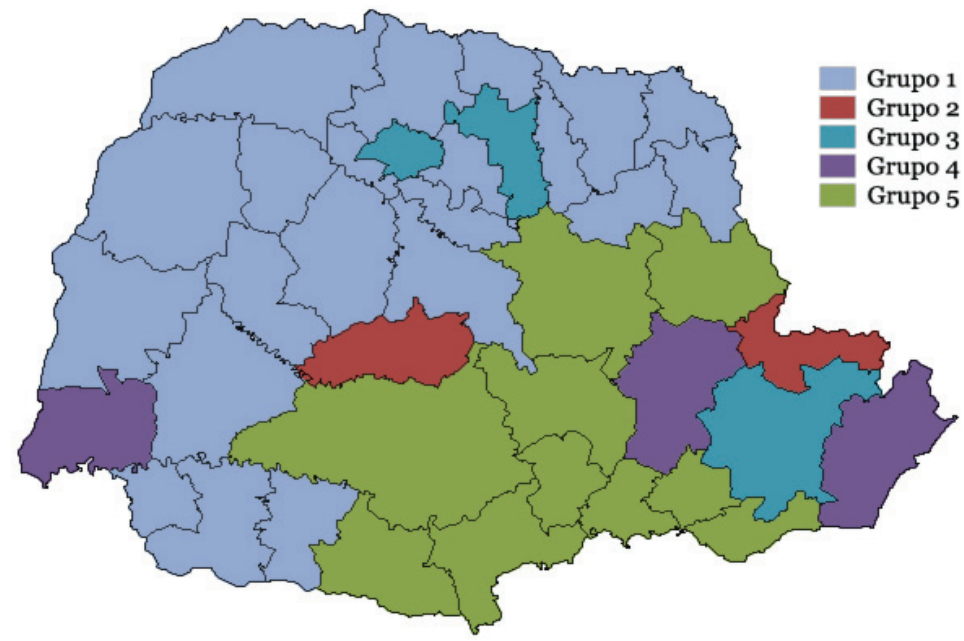

FONTE: resultados de pesquisa. Adaptado de Ipardes (2012).

Os valores médios dos indicadores (escores fatoriais) dos grupos, apresentados na Tabela 7, possibilitam uma comparação entre os níveis de desenvolvimento de cada um deles. No sentido de melhor ilustrar tais indicadores, estão destacados em negrito os valores máximos e mínimos dos escores para cada fator. Com base nesses resultados, pode-se dizer que o Grupo 2 foi, sem dúvidas, aquele que apresentou as piores condições de desenvolvimento socioeconômico, pois foi responsável pelo pior escore dos Fatores 1 e 3, e ainda esteve entre os piores grupos em relação ao Fator 2. Tal resultado confirma a análise descritiva das variáveis (seção 2.3), onde foi demonstrado que as microrregiões desse grupo (Cerro Azul e Pitanga) apresentam os piores indicadores para quase todos os aspectos considerados neste estudo.

Todos os demais grupos tiveram resultados negativos em apenas um dos três fatores considerados. O Grupo 1, que possui 21 microrregiões, apresentou resultado negativo para desenvolvimento industrial $\left(\mathrm{F}_{3}\right)$, mas teve resultado favorável para o fator relacionado à precariedade dos serviços de saúde (F2) e o melhor indicador de condições de moradia (F1).

O terceiro grupo, apesar dos bons indicadores para os Fatores 1 e 3, foi o pior com relação a urbanização associada à precariedade dos serviços de saúde. Esse grupo, formado por Curitiba, Londrina e Maringá, refere-se a municípios com elevado grau de desenvolvimento, porém com restrições na oferta de serviços básicos (como os de saúde) que estão relacionadas, entre outros aspectos, com o tamanho de suas populações. 
TABELA7.ESCORESFATORIAISMÉDIOSPARAOSGRUPOSDEMICRORREGIÕES

\begin{tabular}{cccc}
\hline Grupo & F1 & F2 & F3 \\
\hline 1 & $\boldsymbol{o , 5 7 7}$ & $-0,163$ & $-0,564$ \\
2 & $\mathbf{- 3 , 1 2 0}$ & 0,718 & $\mathbf{- 1 , 5 3 5}$ \\
3 & 0,058 & $\mathbf{2 , 7 8 7}$ & 0,650 \\
4 & 0,203 & 0,647 & $\mathbf{1 , 5 3 7}$ \\
5 & $-0,666$ & $\mathbf{- o , 8 3 3}$ & 0,836 \\
\hline
\end{tabular}

FONTE: resultados de pesquisa.

O Grupo 4 é aquele com melhor condição de desenvolvimento industrial associado a melhores níveis de educação, além de ter situação favorável de moradias, embora desfavorável com relação a serviços de saúde. Destacamse, nesse conjunto de microrregiões, Ponta Grossa e Paranaguá, as quais concentram boa parte da produção industrial do estado (IPARDES, 2004). Por fim, as melhores condições para oferta de serviços de saúde encontram-se no Grupo 5, que também apresentou indicador favorável de desenvolvimento industrial, mas negativo para condições de moradia de sua população.

\section{Considerações Finais}

Buscou-se neste trabalho avaliar as diferenças de nível de desenvolvimento socioeconômico entre as microrregiões do Estado do Paraná, bem como hierarquizá-las e agrupá-las de acordo com suas características.

Os principais resultados mostraram que, apesar de se tratar de um dos estados mais ricos do Brasil, o Paraná apresenta disparidades regionais consideráveis. Considerando os indicadores de condições de moradia, de precariedade dos serviços de saúde e de desenvolvimento industrial, observou-se que para cada um deles existia uma parcela de microrregiões com boas condições e outra, também de proporção significativa, com péssimos resultados.

A análise de cluster distribuiu as microrregiões em cinco grupos, sendo que o Grupo 2, formado por Cerro Azul e Pitanga, foi aquele com as piores condições de desenvolvimento, a partir dos três indicadores utilizados. Todos os demais grupos apresentaram resultado negativo em apenas um dos três indicadores. Desse modo, observa-se que em todo o Estado do Paraná, por mais que existam certas condições favoráveis, coexistem carências extremamente importantes e que impactam negativamente milhares de famílias.

Esse desenvolvimento desequilibrado, sobretudo no que diz respeito às condições de moradia e infra-estrutura de saúde, requer a formulação de políticas públicas que amenizem essas divergências e que possam proporcionar melhores condições de vida à população paranaense. 


\section{Referências}

FERNANDES, E. A.; CUNHA, N. R. S.; SILVA, R. G. (2005). "Degradação ambiental no Estado de Minas Gerais”. Revista de Economia e Sociologia Rural, vol. 43(1).

FERNAU, M. E.; SAMSON, P. J. (1990). "Use of cluster analysis to define periods of similar meteorology and precipitation chemistry in Eastern North America. Part I: transport patterns”. Journal of Applied Meteorology, vol. 29.

FERREIRA JUNIOR, S.; BAPTISTA, A. J. M. S.; LIMA, J. E. (2004). “A modernização agropecuária nas microrregiões do Estado de Minas Gerais”. Revista de Economia e Sociologia Rural, vol. 42(1).

GUALDA, N. L. P.; TAVARES, A. Z. (2003). “As transformações da economia paranaense como determinante de sua nova base produtiva”. Seminário Itinerante de Economia Paranaense. Toledo, Paraná.

HADDAD, P. R. et al. (1989). Economia regional: teorias e métodos de análise. Fortaleza: BNB ETENE.

HOFFMANN, R. (1992). "A dinâmica da modernização da agricultura em 157 microrregiões homogêneas do Brasil”. Revista de Economia e Sociologia Rural, vol. $30(4)$.

IBGE (2000). Censo Demográfico - 200o. Inst. Brasileiro de Geografia e Estatística. URL [on-line]: <http://www.sidra.ibge.gov.br>. Acesso em: mar. 2010.

IPARDES (2004). Leituras Regionais: mesorregiões geográficas do Paraná. Instituto Paranaense de Desenvolvimento Econômico e Social. Curitiba.

IPARDES (2012). Identificação, caracterização, construção de tipologia e apoio na formulação de políticas para os arranjos produtivos locais (APLs) do Estado do Paraná. Instituto Paranaense de Desenvolvimento Econômico e Social. URL [on-line]: <http://www.ipardes.gov.br>. Acesso em: jun. 2012.

JOHNSON, A.; WICHERN, D. (1988). Applied multivariate statistical analysis. New Jersey.

LEITE, G. B.; BRIGATTE, H.; AGUILAR, E. B. (2009). "Análise multivariada de indicadores socioeconômicos dos países do G-20”. Revista de Economia Mackenzie, vol. 7(1).

MELO, C. O.; PARRÉ, J. L. (2006). “Determinantes do Desenvolvimento Rural dos Municípios da Região Sudoeste Paranaense”. Revista Faz Ciência, vol. 8(1).

MELO, C. O.; PARRÉ, J. L. (2007). "Índice de Desenvolvimento Rural dos Municípios Paranaenses: Determinantes e Hierarquização”. Revista de Economia e Sociologia Rural, vol. 45(2).

Mingoti, S. A. (2007). Análise de Dados Através de Métodos de Estatística Multivariada. Editora UFMG. 
PEROBELli, F. S.; OLIVEIRA, A. F.; NOVY, L. G. G.; FERREIRA, M. V. (1999). "Planejamento regional e potenciais de desenvolvimento dos municípios de Minas Gerais na região em torno de Juiz de Fora: aplicação de análise fatorial”. Nova Economia, vol. 9(1).

PNUD (2010). Atlas do Desenvolvimento Humano. Programa das Nações Unidas para o Desenvolvimento. URL [on-line]: <http://www.pnud.org.br/atlas/> Acesso em: mar. 2010.

REIS, J. N. ; LIMA, P. H. (1995). “Desenvolvimento sócio-econômico e hierarquização dos municípios cearenses”. Revista Econômica do Nordeste, vol. 26(4).

REZENDE, L. P. F.; PARRÉ, J. L. (2003). “Comparação do grau de desenvolvimento agrícola dos municípios paranaenses”. Congresso da Sociedade Brasileira de Economia e Sociologia Rural, 41. Juiz de Fora.

REZENDE, L. P. F.; PARRÉ, J. L. (2004). “A regionalização da agricultura paranaense na década de noventa: um estudo utilizando estatística multivariada”. Congresso da Sociedade Brasileira de Economia e Sociologia Rural, 42, Cuiabá.

ROSADO, P. L.; ROSSATO, M. V.; LIMA, J. E. (2009). “Análise do Desenvolvimento Socioeconômico das Microrregiões de Minas Gerais”. Revista Econômica do Nordeste, vol. 40(2).

SILVA, R. G.; RIBEIRO, C. G. (2004). “Análise da degradação ambiental na Amazônia Ocidental: um estudo de caso dos municípios do Acre”. Revista de Economia e Sociologia Rural, vol. 42(1).

SILVA, V. M. (1995). "A distribuição dos recursos para os municípios paulistas: uma abordagem de análise multivariada”. Estudos Econômicos, vol. 25(3).

SOARES, A. C. L. G. et al. (1999). "Índice de desenvolvimento municipal: hierarquização dos municípios do Ceará no ano de 1997”. Revista Paranaense de Desenvolvimento, n. ${ }^{\circ} 97$.

TAVARES, J. M. (2008). Identificação de Padrões Distintos de Desenvolvimento na Região Sul do Brasil: uma análise multivariada. Tese de Doutorado. Programa de Pós-Graduação em Economia da Faculdade de Ciências Econômicas. Universidade Federal do Rio Grande do Sul (UFRGS).

TAVARES, J. M.; PORTO JUNIOR, S. S. (2008) "Desigualdades intra e interregionais em Santa Catarina: uma análise multivariada”. Encontro Regional de Economia ANPEC Sul, 11. Curitiba.

Artigo recebido em: 21 de agosto de 2011

Aceito em: 17 de agosto de 2012 


\section{Anexos}

\section{FIGURA A1. MICRORREGIÕES GEOGRÁFICAS DO ESTADO DO PARANÁ}

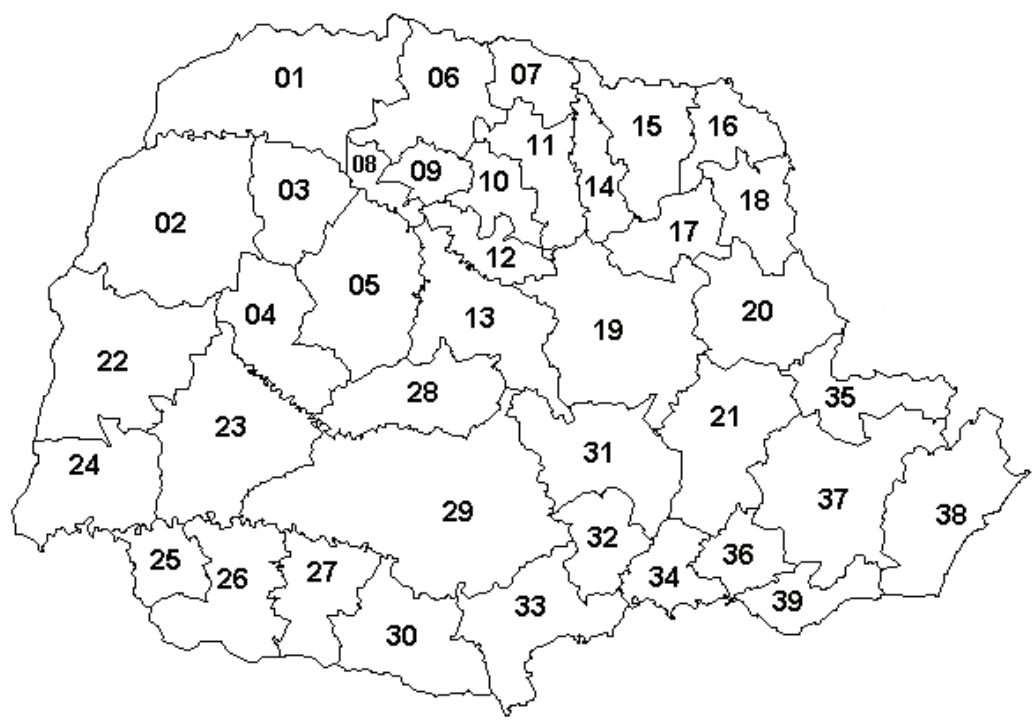

FONTE: adaptado de Ipardes (2012).

Nota: A correspondência se dá por 01 - Paranavaí, 02 - Umuarama, 03 - Cianorte , 04 - Goiorê, 05 - Campo Mourão, o6 - Astorga, 07 - Porecatu, 08 - Floraí, o9 - Maringá, 10 - Apucarana, 11 - Londrina, 12 - Faxinal, 13 - Ivaiporã, 14 - Assaí, 15 - Cornélio Procópio, 16 - Jacarezinho, 17 - Ibaiti, 18 - Wesceslau Braz, 19 - Telêmaco Borba, 20 - Jaguariaiva, 21 - Ponta Grossa, 22 - Toledo, 23 - Cascavel, 24 - Foz do Iguaçú, 25 - Capanema, 26 - Francisco Beltrão, 27 - Pato Branco, 28 - Pitanga, 29 - Guarapuava, 30 - Palmas , 31 - Prudentópolis, 32 - Irati, 33 - União da Vitória, 34 - São Mateus do Sul, 35 - Cerro Azul, 36 - Lapa, 37 - Curitiba, 38 Paranaguá, 39 - Rio Negro. 
VIDIGAL, V. G.; AMARAL, I. C.; SILVEIRA, G. F. Desenvolvimento socioeconômico nas microrregiões...

TABELA A1. RESULTADOS DO TESTE DE KAISER-MEYER-OLKIN (KMO) PARA AS VARIÁVEIS SOCIOECONÔMICAS OBSERVADAS NAS MICRORREGIÕES PARANAENSES

\begin{tabular}{lc}
\hline Variável & Estatística KMO \\
\hline analf & 0,800 \\
clixo & 0,811 \\
sagua & 0,726 \\
sban & 0,681 \\
rendmin & 0,703 \\
gelad & 0,807 \\
ilumin & 0,747 \\
comput & 0,814 \\
tel & 0,842 \\
telev & 0,823 \\
leit & 0,575 \\
hosp & 0,603 \\
ddemog & 0,799 \\
urban & 0,868 \\
morad & 0,828 \\
mort & 0,356 \\
pibagro & 0,642 \\
pibind & 0,445 \\
pibserv & 0,452 \\
pibcapita & 0,670 \\
\hline Geral & 0,724 \\
\hline
\end{tabular}

FONTE: resultados de pesquisa. 
VIDIGAL, V. G.; AMARAL, I. C.; SILVEIRA, G. F. Desenvolvimento socioeconômico nas microrregiões...

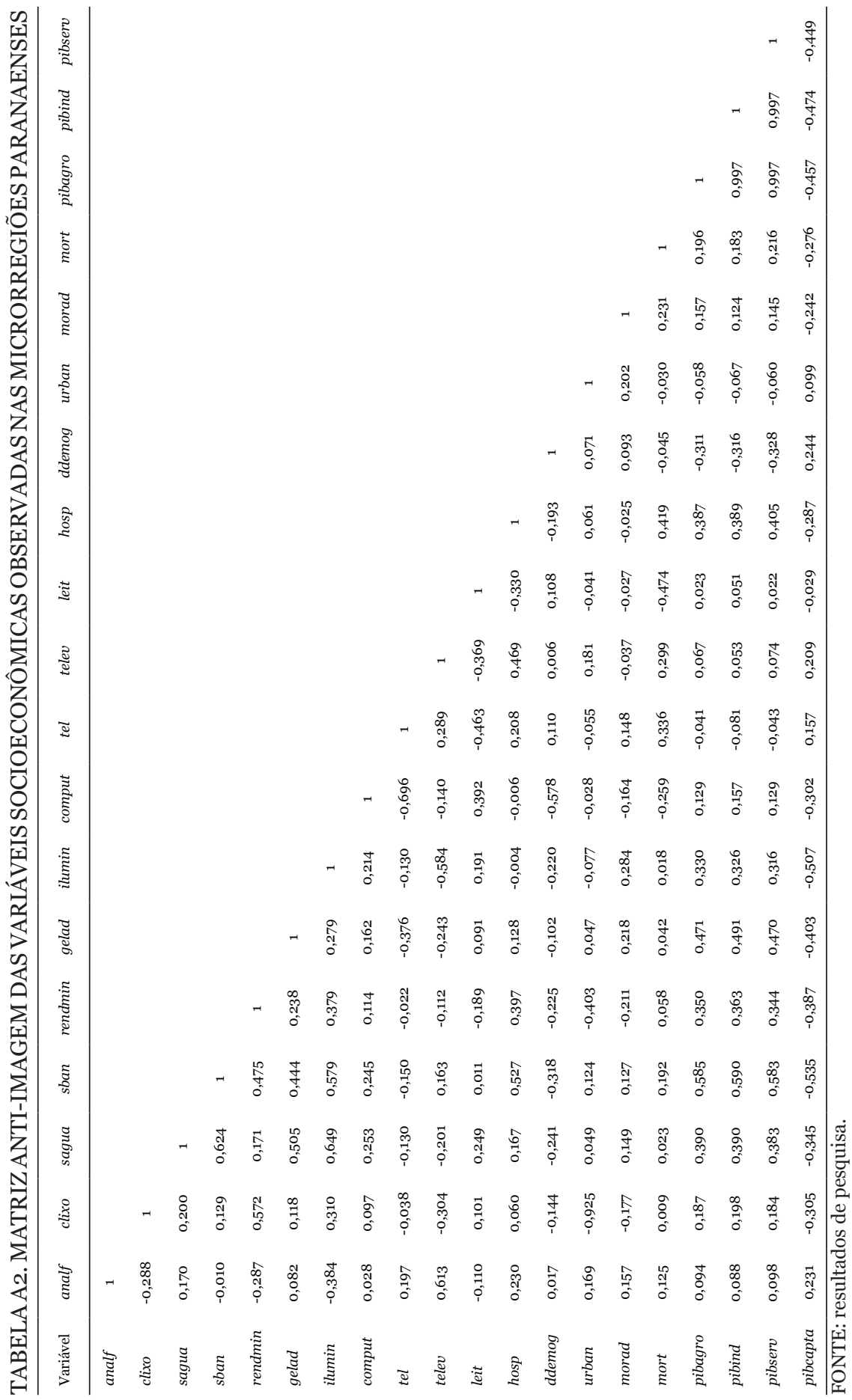

